\title{
CEM - A Program for Visualization and Discovery in Email
}

\author{
Richard Cole $^{1}$, Peter Eklund ${ }^{1}$, Gerd Stumme $^{2}$ \\ ${ }^{1}$ Knowledge, Visualization and Ordering Laboratory \\ School of Information Technology, Griffith University, AUSTRALIA \\ r.cole,p.eklund@gu.edu.au \\ ${ }^{2}$ Institut für Angewandte Informatik und Formale \\ Beschreibungsverfahren, Universität Karlsruhe (TH), GERMANY \\ stumme@aifb.uni-karlsruhe.de
}

\begin{abstract}
This paper presents a lattice metaphor for knowledge discovery in electronic mail. It allows a user to navigate email using a lattice rather than a tree structure. By using such a conceptual multi-hierarchy, the content and shape of the lattice can be varied to accommodate queries against the email collection. This paper presents the underlying mathematical structures, and a number of examples of the lattice and multihierarchy working with a prototypical email collection.
\end{abstract}

\section{Introduction}

Email management systems usually store email as a tree: in analogue to the file management system. This has an advantage in that trees are easily explained to novice users as a direct mapping from the physical structure of the file system. The disadvantage is that, when the email is stored, the user preempts the way he will later retrieve the email. The tree structure forces a decision about the criteria considered as primary and secondary indexes for the email. For instance, when storing email regarding the organization of a conference, one needs to decide whether to organise the email as Komorowski/pkdd2000/program_committee where "Komorowski" is a primary index alternatively conferences/pkdd/pkdd2000/ organization. This problem is common when a user cooperates with overlapping subjects with different topics and multiple views.

This paper profiles a Conceptual Email Manager called CEM. This follows earlier work $[3,2]$. CEM is a lattice-based email retrieval and storage program that aids knowledge discovery by using flexible views over email. It uses a concept lattice-based data structure for storing emails rather than a tree. This permits clients to retrieve emails along different paths. In the example above, the client need not decide which path to store the email. When retrieving the email later, he can consider any combination of the catchwords in the two paths. Email retrieval is totally independent of the physical organization of the file system.

Related approaches to the above problem include the idea of a virtual folder that was introduced in a program called View Mail (VM)[6]. A virtual folder is a collection of email documents retrieved in response to a query. The virtual folder

D.A. Zighed, J. Komorowski, and J. Zytkow (Eds.): PKDD 2000, LNAI 1910, pp. 367-374, 2000.

(C) Springer-Verlag Berlin Heidelberg 2000 
concept has more recently been popularised by a number of open-source projects, e.g. [8]. Our system differs from those projects in the understanding of the underlying structure - via formal concept analysis - and in its implementation.

Concept lattices are defined in the mathematical theory of Formal Concept Analysis [11]. A concept lattice derives from a binary relation assigning attributes to objects. In our application, the objects are all emails stored by the system, and the attributes catchwords like 'conferences', 'Komorowski', or 'organization'. We assume the reader to be familiar with the basic notions of Formal Concept Analysis, and otherwise refer to [5]. In the next section, we describe the mathematical structures of the CEM. Requirements for their maintenance are discussed in Section 3. We also describe how they are fulfilled by our implementation.

\section{Mathematical Structure}

In this section we describe the system on a structural level. We distinguish three fundamental structures: (i) a formal context assigning to each email a set of catchwords; (ii) a hierarchy on catchwords to define more general catchwords; (iii) creating conceptual scales as a graphical interface for email retrieval.

In CEM, we use a formal context $(G, M, I)$ for storing email and assigning catchwords. $G$ is a set containing all emails, the set $M$ contains all catchwords. For the moment, we consider $M$ to be unstructured (we will subsequently introduce a hierarchy on it.) The relation $I$ indicates emails assigned to catchwords. In the previous example, the client might assign the catchwords 'Komorowski', 'pkdd2000, 'program_committee', 'conferences', 'pkdd, and 'organization' to a new email. The incidence relation is generated in a semi-automatic process: (i) an regular-expression string-search algorithm recognizes words within an email suggesting relations between email attributes, (ii) the client may accept the string-search result or otherwise modify it, and (iii) the client may attach his own attributes to the email.

Instead of a tree of disjoint folders and sub-folders, we consider the concept lattice $\underline{\mathfrak{B}}(G, M, I)$ as navigation space. The formal concepts replace the folders. In particular, this means emails may appear in different concepts. The most general concept contains all email. The deeper the client moves into the hierarchy, the more specific the concepts, and the fewer emails they contain.

To support the semi-automatic assignment of catchwords to the emails, we provide the set $M$ of catchwords with a partial order $\leq$. For this subsumption hierarchy we assume that a compatibility condition holds: $\forall g \in G, m, n \in$ $M: \quad(g, m) \in I, m \leq n \Rightarrow(g, n) \in I \quad(\ddagger)$ i.e., the assignment of catchwords respects the transitivity of the partial order. Hence, when assigning catchwords to emails, it is sufficient to assign only the most specific catchwords. More general catchwords are automatically added.

For instance, the user may want to say that 'pkdd' is more specific than 'conferences', and 'pkdd2000' more specific than 'pkdd (i. e., 'pkdd2000' $\leq$ 'pkdd' $\leq$ 'conferences'). Emails about this paper are assigned by the client to 'pkdd2000' only (as well as any additional catchwords like 'cole', 'eklund' and 'stumme'). When the email-client retrieves this email, no pathname is recalled. Instead, the 
emails appear under the more general catchword 'conferences'. If 'conferences' provides too large a list of email, the client can refine the search by choosing a sub-term like 'pkdd', or adding a new catchword, for instance 'cole'.

Note that even though we impose no specific structure on the subsumption hierarchy $(M, \leq)$ it naturally splits three ways. One relates the contents of the emails. A second to the sender or receiver of the email and the third describes the emailing process (in-bound or out-bound). An example of a hierarchy is given in Fig. 1 which is a forest (i. e., a union of trees), but the resulting concept lattice - used as the search space - is by no means a forest. The partially order set is displayed both in the style of a folding editor and as a connected graph.

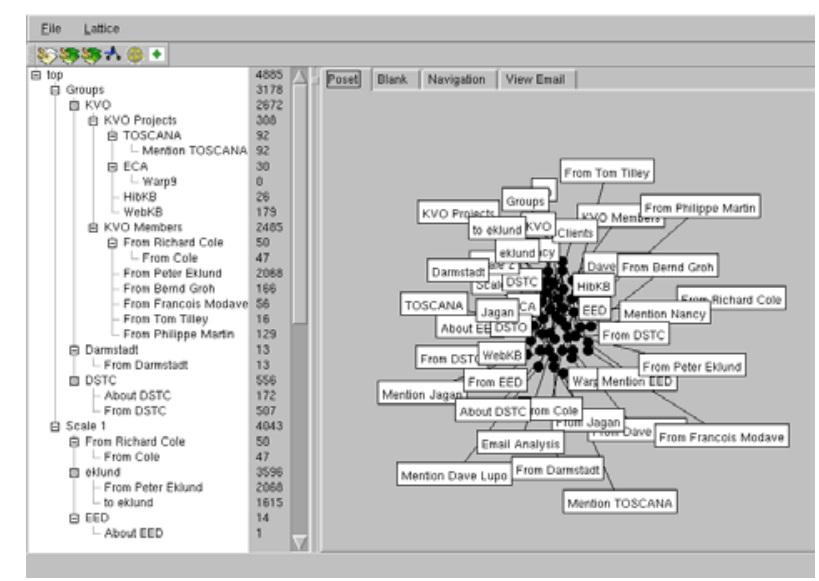

Fig. 1. Partially ordered set of catchwords: as a folding editor and connected graph.

Consider the concept generated by the conjunction of the two catchwords 'PKDD 2000' and 'conference organization'. It will have at least two incomparable super-concepts, one generated by the catchword 'PKDD 2000' and another generated by 'conference organization'. In general, the resulting concept lattice is embedded as a join-semilattice in the lattice of all order ideals (i. e., all subsets $X \subseteq M$ s.t. $x \in X$ and $x \leq y$ imply $y \in X)$ of $(M, \leq)$.

Conceptual scaling deals with many-valued attributes. Often attributes are not one-valued but allow a range of values. This is modelled by a many-valued context. A many-valued context is roughly equivalent to a relation in a relational database with one field being a primary key. As one-valued contexts are special cases of many-valued contexts, conceptual scaling can also be applied to onevalued contexts to reduce the complexity of the visualization (readers who are interested in the exact definition of many-valued contexts and the use of conceptual scaling in general are referred to [5]). Applied to one-valued contexts, conceptual scales determine the concept lattice that arises from one vertical 'slice' of a large context: 
Definition 1. A conceptual scale for a subset $B \subseteq M$ of attributes is a (onevalued) formal context $\mathbb{S}_{B}:=\left(G_{B}, B, \ni\right)$ with $G_{B} \subseteq \mathfrak{P}(B)$. The scale is called consistent wrt $\mathbb{K}:=(G, M, I)$ if $\{g\}^{\prime} \cap B \in G_{B}$ for each $g \in G$. For a consistent scale $\mathbb{S}_{B}$, the context $\mathbb{S}_{B}(\mathbb{K}):=(G, B, I \cap(G \times B))$ is called its realized scale.

Conceptual scales group together related attributes. They are determined by the user, and the realized scales derived from them when a diagram is requested. CEM stores all scales defined by the client in previous sessions. The client assigns to each scale a unique name. This is modelled by a mapping $(\mathcal{S})$.

Definition 2. Let $\mathcal{S}$ be a set whose elements are called scale names. The mapping $\alpha: \mathcal{S} \rightarrow \mathfrak{P}(\mathcal{M})$ defines for each scale name $s \in \mathcal{S}$ a scale $\mathbb{S}_{s}:=\mathbb{S}_{\alpha(s)}$.

For instance, the user may introduce a new scale that classifies emails according to being related to a conference by adding a new element 'Conference' to $\mathcal{S}$ and by defining $\alpha$ (Conference) $:=\{$ CKP'96, AA 55, KLI ‘98, Wissen '99, PKDD 2000 .

Observe that $\mathcal{S}$ and $M$ need not be disjoint. This allows the following construction deducing conceptual scales directly from the subsumption hierarchy: Let $\mathcal{S}:=\{m \in M \mid \exists n \in M: n<m\}$, and define, for $s \in \mathcal{S}, \alpha(s):=\{m \in M \mid m \prec$ $s\}$ (with $x \prec y$ if and only if $x<y$ and there is no $z$ s.t. $x<z<y$ ). This means all catchwords $m \in M$, neither minimal nor maximal in the hierarchy, are considered as the name of scale $\mathbb{S}_{m}$ and as a catchword of another scale $\mathbb{S}_{n}$ (where $m \prec n$ ). This last construction, presented in [9], defines a hierarchy of conceptual scales for a library information system [7].

\section{Requirements of the CEM}

We now discuss the requirements of the CEM based on the Formal Concept Analysis. We explain how our implementation responds to these requirements. Requirements are divided along the same lines as the mathematical structures defined in Section 2; (i) assist the client edit and browse a catchword hierarchy; (ii) help visualize and modify the scale function $\alpha$; (iii) allow the client to manage the assignment of catchwords to emails; (iv) assist the client search the space of emails for individual emails and conceptual groupings.

In addition to the requirements stated above, a good email system needs to be able send, receive and display emails: processing various email formats and interacting with popular protocols which are well understood and implemented in existing email programs so not discussed further.

The catchword hierarchy is a partially ordered set $(M, \leq)$ where each element of $M$ is a catchword. The requirements for editing and browsing the catchword hierarchy are: (i) graphically display the structure of the $(M, \leq)$. The ordering relation must be evident to the client; (ii) make accessible to the client a series of direct manipulations to alter the ordering relation. It should be possible to create any partial order to a reasonable size limit.

The user must be able to visualize the scale function, $\alpha$, explained in Section 2. The program must allow an overlap between the set of scale labels, $\mathcal{S}$, and the set of catchwords $M$. Section 2 introduced the formal context $(G, M, I)$. 


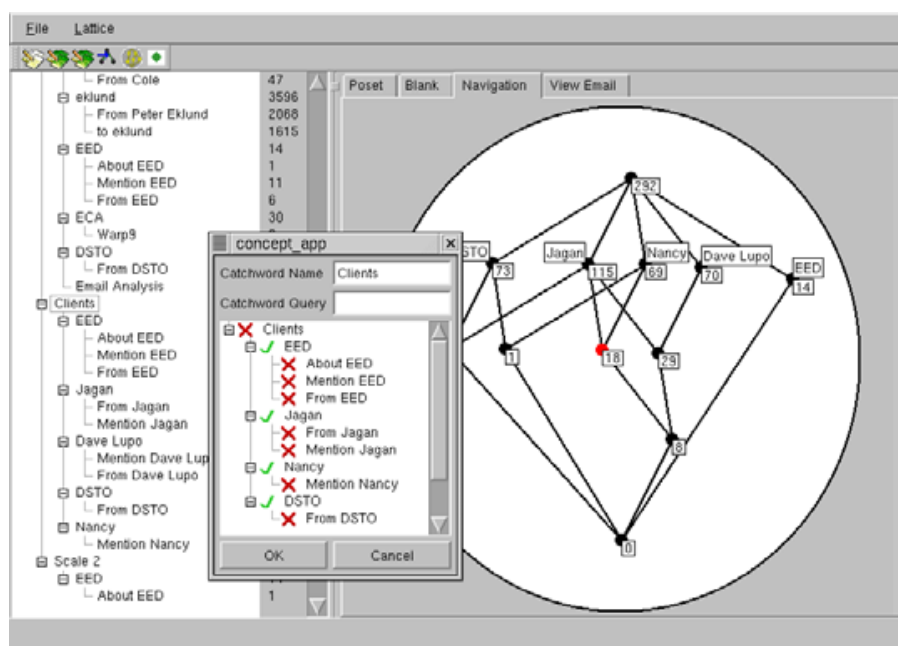

Fig. 2. Scale, catchword and concept lattice. The central dialogue box shows how $\alpha$ can be edited.

This formal context associates email with catchwords via the relation $I$. Also introduced was the notion of the compatability condition, $(\ddagger)$.

The program should store the formal context $(G, M, I)$ and ensure the compatability condition is always satisfied. It is inevitable that the program will have to sometimes modify the formal context in order to satisfy the compatability condition after a change is made to the catchword hierarchy. The program must support two mechanisms for the association of catchwords to emails. Firstly, a mechanism where emails are automatically associated with catchwords based on the email content. Secondly, the client the should be able to view and modify the association of catchwords with emails. The program must allow the navigation of the conceptual space of the emails by drawing line diagrams of concept lattices derived from conceptual scales [5]. This is shown in Fig. 2. These line diagrams should extend to locally scaled nested line diagrams[9] shown in Fig. 3. The program must allow retrieval and display of emails forming the extension of concepts displayed in the line diagrams.

\section{Implementation of CEM}

This section divides the description of implementation of the CEM into a similar structure to that presented in Section 3. The user is presented with a view of the hierarchy, $(M, \leq)$ as a tree widget $^{1}$, shown in Fig. 2. The catchword hierarchy, being a partially ordered set, is a more general structure than a tree. Although the example given in Fig. 1 is a forest, no limitation is placed by the program on the structure of the partial order other than as a partial order.

The following is a definition of a tree derived from the catchword hierarchy for the purpose defining the contents and structure of the tree widget. Let $(M, \leq)$

$\overline{1}$ A widget is a graphical user interface component with a well defined behaviour usually mimicking some physical object, for example a button. 


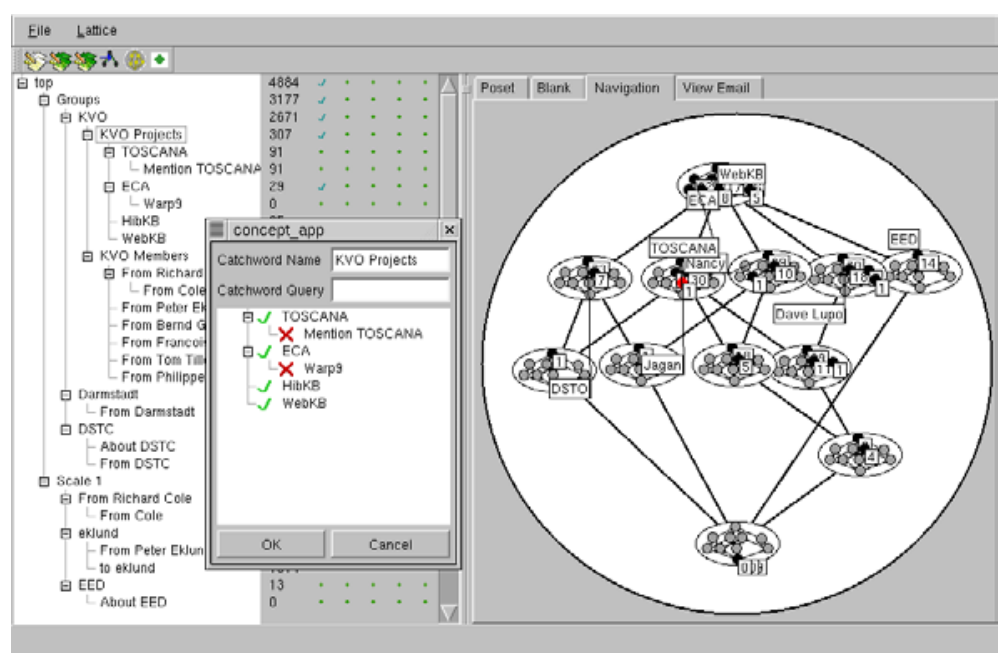

Fig. 3. Scale, catchword and nested-line diagram.

be a partially ordered set and denote the set of all sequences of elements from $M$ by $\langle M>$. Then the tree derived from the catchword hierarchy is comprised by ( $T$, parent, label), where $T \subseteq<M>$ is a set of tree nodes, $<>$ the empty sequence is the root of the tree, parent $: T /<>\rightarrow T$ is a function giving the parent node of each node (except the root node), and label : $T \rightarrow M$ assigns a catchword to each tree node.

$$
\begin{aligned}
T=\left\{<m_{1}, \ldots, m_{n}>\in<M>\mid\right. & \left.m_{i} \preceq m_{i+1} \text { and } m_{n} \in \operatorname{top}(M)\right\} \\
\operatorname{parent}\left(<m_{1}, \ldots, m_{n}>\right) & :=<m_{1}, \ldots, m_{n-1}> \\
\operatorname{parent}\left(<m_{1}>\right) & :=<> \\
\operatorname{label}\left(<m_{1}, \ldots, m_{n}>\right) & :=m_{1}
\end{aligned}
$$

Each tree node is identified by a path from a catchword to the top of the catchword hierarchy. The tree representation has the disadvantage that elements from the partial order occur multiple times in the tree and the tree can become large. If however the user keeps the number of elements with multiple parents in the partial order to a small number, the tree is manageable.

The program provides four operations for modifying the hierarchy: insert \& remove catchword and insert \& remove ordering. More complex operations provided to the client, moving an item in the taxonomy, are resolved internally to a sequences of these basic operations. In this section we denote the order filter of $m$ as $\uparrow m:=\{x \in M \mid m \leq x\}$, the order ideal of $m$ as $\downarrow m:=\{x \in M \mid x \leq m\}$, and the upper cover of $m$ as $\succ_{m}:=\{x \in M \mid x \succ m\}$.

The operation of insert catchword simply adds a new catchword to $M$, and leaves the $\leq$ relation unchanged. The remove catchword operation takes a single parameter $a \in M$ for which the lower cover is empty, and simply removes $a$ from $M$ and $(\uparrow a) \times\{a\}$ from the ordering relation.

The operation of insert ordering takes two parameters $a, b \in M$ and inserts into the relation $\leq$, the set $(\uparrow a) \times(\downarrow b)$. The operation of remove ordering takes 
two parameters $a, b \in M$ where $a$ is an upper cover of $b$. The remove ordering operation removes from $\leq$ the set $\left(\left(\uparrow a / \uparrow\left(\succ_{b} / a\right)\right) \times(\downarrow b)\right)$.

The set of scales $S$, according to the mathematization in Section 2 is not disjoint from $M$, thus the tree representation of $M$ already presents a view of a portion of $S$. In order to reduce the complexity of the graphical interface, we make $S$ equal to $M$, i.e. all catchwords are scale labels, and all scale labels are catchwords. The function $\alpha$ maps each catchword $m$ to a set of catchwords. The program displays this set of catchwords, when requested by the user, using a dialog (see Fig. 2 - centre). The dialog box contains all catchwords in the downset of $m$ an icon (either a tick, or a cross) to indicate membership in the set of catchwords given by alpha $(m)$. Clicking the icon changes $\alpha(m)$ 's membership.

By only displaying the down-set of $m$ in the dialog box, the program restricts the definition of $\alpha$ to $\alpha(m) \subseteq \downarrow m$. This has an effect on the "remove ordering operation" defined on $(M, \leq)$. When the ordering of $a \leq b$ is removed the image of $\alpha$ function for attributes in $\uparrow a$ must be checked and possibly modified.

Each member of $(M, \leq)$ is associated with a query term, in this application is a set of section/word pairs. That is: Let $H$ be the set of sections found in the email documents, $W$ the set of words found in email documents, then a function query: $M \rightarrow \mathfrak{P}(H \times W)$ attaches to each attribute a set of section/word pairs.

Let $G$ be a set of email. An inverted file index stores a relation $R_{1} \subseteq G \times$ $(H \times W)$ between documents and section/word pairs. $(g,(h, w)) \in R_{1}$ indicates that document $g$ has word $w$ in section $h$.

A relation $R_{2} \subseteq G \times M$ is derived from the relation $R_{1}$ and the function query via: $(g, m) \in R_{2}$ iff $(g,(h, w)) \in R_{1}$ for some $(h, w) \in$ query $(m)$. A relation $R_{3}$ stores user judgements saying that an email should have an attribute $m$. A relation $R_{4}$ respecting the compatibility condition $(\ddagger)$ is then derived from the relations $R_{2}$ and $R_{3}$ via: $(g, m) \in R_{4}$ iff there exists $m_{1} \leq m$ with $\left(g, m_{1}\right) \in R_{2} \cup R_{3}$.

Inserting the ordering $b \leq a$ into $\leq$ requires the insertion of set $(\uparrow a / \uparrow$ b) $\times\left\{g \in G \mid(g, b) \in R_{4}\right\}$ into $R_{4}$. Such an insertion into an inverted file index is $O(n m)$ where $n$ is the average number of entries in the inverted index in the shaded region, and $m$ is the number of elements in the shaded region. The real complexity of this operation is best determined via experimentation with a large document sets and a large user defined hierarchy [1]. Similarly the removal of the ordering $b \leq a$ from $\leq$ will require a re-computation of the inverted file entries for elements in $\uparrow a$.

When new emails, $G_{b}$, are presented to CEM, the relation $R_{1}$ is updated by inserting new pairs, $R_{1 b}$, into the relation. The modification of $R_{1}$ into $R_{1} \cup R_{1 b}$ causes an insertion of pairs $R_{2 b}$ into $R_{2}$ according to query $(m)$ and then subsequently an insertion of new pairs $R_{4 b}$ into $R_{4}$.

$$
\begin{aligned}
& R_{1 b} \subseteq G_{b} \times(H \times W) \\
& R_{2 b}=\left\{(g, m) \mid \exists(h, w) \in \operatorname{query}(m) \text { and }(g,(h, w)) \in R_{1 b}\right\} \\
& R_{4 b}=\left\{(g, m) \mid \exists m_{1} \leq m \text { with }\left(g, m_{1}\right) \in R_{2 b}\right\}
\end{aligned}
$$

When the user makes a judgement that an indexed email should be associated with an attribute, $m$, then an update must be made to $R_{3}$, which will in turn 
cause updates to all attributes in the order filter of $m$ to be updated in $R_{4}$. In the case that a client retracts a judgement, saying that an email is no longer be associate with an attribute, $m$, requires a possible update to each attribute, $n$, in the order filter of $m$.

When the user requests that the concept lattice derived from the scale with name $s \in S$ be drawn, the program computes $\mathbb{S}_{\alpha(S)}$ from Definition 1 via the algorithm reported in [1]. In the case that the user requests a diagram combining two scales with names labels $s$ and $t$, then the scale $\mathbb{S}_{B \cup C}$ with $B=\alpha(s)$ and $C=\alpha(t)$ is calculated by the program and its concept lattice $\underline{\mathfrak{B}}\left(\mathbb{S}_{B \cup C}\right)$ is drawn as a projection into the lattice product $\underline{\mathfrak{B}}\left(\mathbb{S}_{B}\right) \times \underline{\mathfrak{B}}\left(\mathbb{S}_{C}\right)$.

\section{Conclusion}

This paper gives a mathematical description of the algebraic structures that can be used to create a a lattice-based view of electronic mail. The claim is that this structure, its implementation and operation, aid the process of knowledge discovery in large collections of email. By using such a conceptual multi-hierarchy, the content and shape of the lattice view is varied. An efficient implementation of the index promotes client iteration.

\section{References}

1. R. Cole, P. Eklund: Scalability in Formal Concept Analysis: A Case Study using Medical Texts. Computational Intelligence, Vol. 15, No. 1, pp. 11-27, 1999.

2. R. Cole, P. Eklund: Analyzing an Email Collection using Formal Concept Analysis. Proc. of the European Conf. on Knowledge and Data Discovery, pp. 309-315, LNAI 1704, Springer, Prague, 1999.

3. R. Cole, P. W. Eklund, D. Walker: Using Conceptual Scaling in Formal Concept Analysis for Knowledge and Data Discovery in Medical Texts, Proceedings of the Second Pacific Asian Conference on Knowledge Discovery and Data Mining, pp. 378-379, World Scientific, 1998.

4. A. Fall: Dynamic taxonomical encoding using sparce terms. 4th Int. Conf. on Conceptual Structures. Lecture Notes in Artificial Intelligence 1115, 1996,

5. B. Ganter, R. Wille: Formal Concept Analysis: Mathematical Foundations. Springer, Heidelberg 1999 (Translation of: Formale Begriffsanalyse: Mathematische Grundlagen. Springer, Heidelberg 1996)

6. K. Jones: View Mail Users Manual. http://www.wonderworks.com/vm. 1999

7. T. Rock, R. Wille: Ein TOSCANA-System zur Literatursuche. In: G. Stumme and R. Wille (eds.): Begriffliche Wissensverarbeitung: Methoden und Anwendungen. Springer, Berlin-Heidelberg 2000

8. W. Schuller: http://gmail.linuxpower.org/. 1999

9. G. Stumme: Hierarchies of Conceptual Scales. Proc. Workshop on Knowledge Acquisition, Modeling and Management. Banff, 16.-22. October 1999

10. F. Vogt, R. Wille: TOSCANA - A graphical tool for analyzing and exploring data. In: R. Tamassia, I. G. Tollis (eds.): Graph Drawing '94, Lecture Notes in Computer Sciences 894, Springer, Heidelberg 1995, 226-233

11. R. Wille: Restructuring lattice theory: an approach based on hierarchies of concepts. In: I. Rival (ed.): Ordered sets. Reidel, Dordrecht-Boston 1982, 445-470 